\title{
Optimizing the detection, ablation, and ion extraction efficiency of a single-particle laser ablation mass spectrometer for application in environments with low aerosol particle concentrations
}

Hans-Christian Clemen et al.

Correspondence to: Johannes Schneider (johannes.schneider@mpic.de)

The copyright of individual parts of the supplement might differ from the CC BY 4.0 License. 


\section{S1 Particle size measurement}

Size-resolved measurements with PSL particles of known size are used to convert the measured particle flight times between the two detection lasers into vacuum aerodynamic diameters at the respective ALS pressure. For this purpose, a corresponding averaged flight time between the first and the second detection laser was determined for each particle size, which results

5 from a time counter of the electronics and is called "upcounts". The upcounts values multiplied by the $40 \mathrm{~ns}$ time steps of the time counter correspond to the flight time of the particles in nanoseconds. Afterwards Eq. S1 was fitted to the size-dependent upcounts values (see Fig. S1). This was performed for different lens pressures, since the particle velocity depends on the pressure gradient at the exit of the ALS. The PSL particles used here are regarded as almost perfect standard calibration particles due to their spherical shape and a particle density of $1.05 \mathrm{~g} \mathrm{~cm}^{-3}$. Using Eq. S1 and the resulting pressure specific curve fit parameters, the particle flight times were converted into vacuum aerodynamic diameters, taking into account the particle density. The correlation between the particle diameters and the determined flight times as upcounts values is shown in Fig. S1.

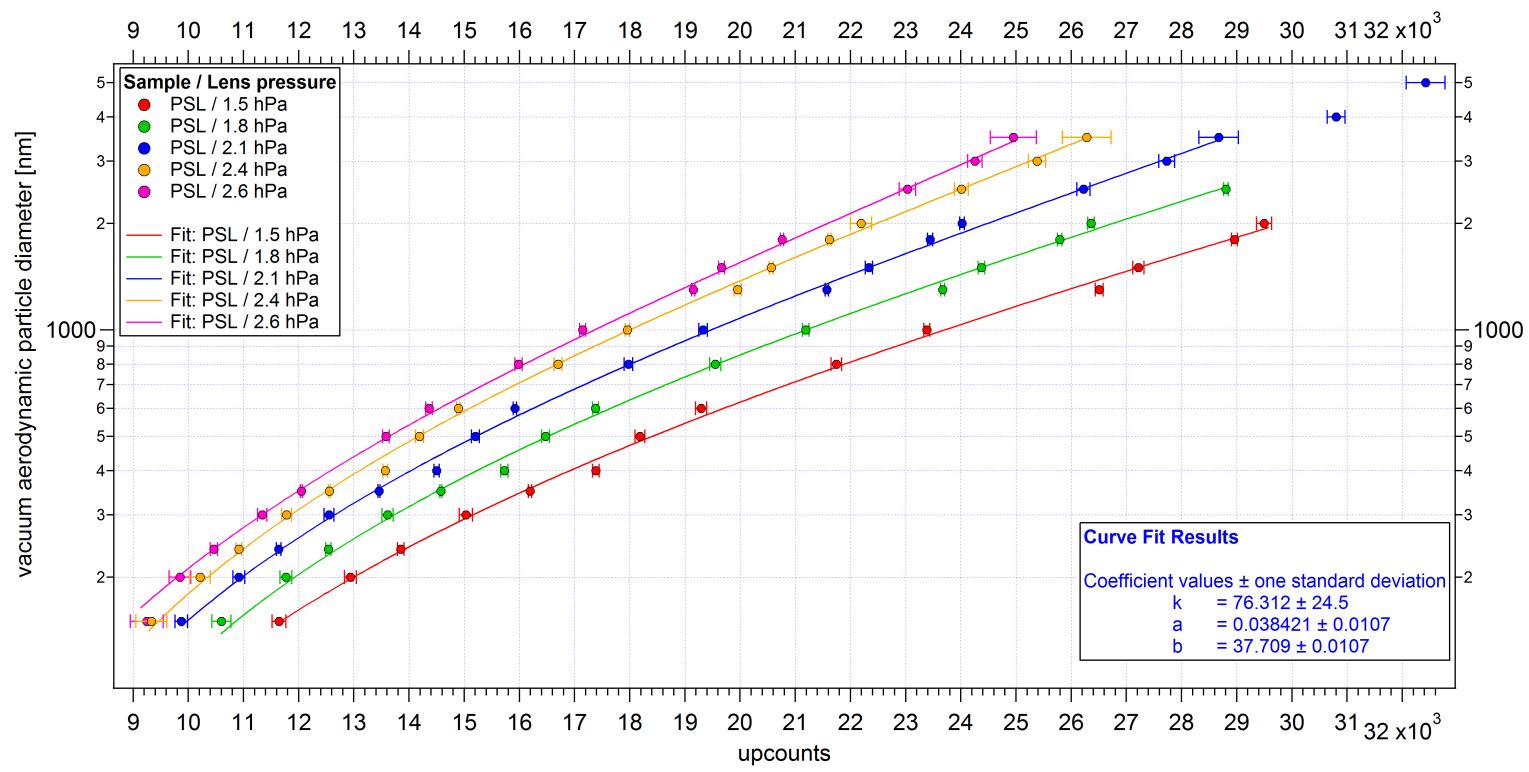

Figure S1. Size calibration curves using PSL particles for lens pressures between $1.5 \mathrm{hPa}$ and $2.6 \mathrm{hPa}$. The curve fit results are obtained for a lens pressure of $2.1 \mathrm{hPa}$. The particle flight times were determined with the ALABAMA including the new ALS. The x-uncertainty bars correspond to the standard deviation of the particle size distribution per particle size, particle type and lens pressure measured with ALABAMA.

The size calibration equation modified according to Klimach (2012):

$$
d_{v a}=\frac{k}{\ln \left(\frac{a \cdot \text { upcounts }-b}{a \cdot \text { upcounts }-\frac{L}{\text { upcounts } \cdot 40 \cdot 10^{-9}}}\right)}
$$

with the dimensionless fit parameters $k, a$ and $b . L$ is kept constant at 0.07 . Due to the modification, the physical relationship of the equation as described in Klimach (2012) and Köllner (2020) is no longer given and must be considered as purely empirical. The adaptation of the equation had become necessary due to the larger particle size range. 
Equation S1 was fitted to the measured points in the particle size range between $150 \mathrm{~nm}$ and $3500 \mathrm{~nm}$. Particle flight times above $3500 \mathrm{~nm}$ were not taken into account because the fit would have been overestimated and could therefore not represent well enough the smaller sizes. Thus, the flight times for the PSL particles at $4170 \mathrm{~nm}$ and $4900 \mathrm{~nm}$ (according to the manufacturer) were not used to determine the size calibration parameters at $2.1 \mathrm{hPa}$. Due to the fact that sizes above $3500 \mathrm{~nm}$ were not taken into account, extrapolation of the calibration curve for these particles ultimately resulted in significantly larger vacuum aerodynamic diameters than would result from calculations (DeCarlo et al., 2004).

\section{S2 Calculations with the aerosol lens calculator}

In the following a brief comparison between the results obtained by the aerosol lens calculator (Wang and McMurry, 2006a, b) for the Liu-type lens and our new aerodynamic lens, focusing on the particle beam width (Fig. S2). For particle sizes below $500 \mathrm{~nm}$, the calculations of the aerosol lens calculator result in a slightly better focusing characteristic of the Liu-type lens compared to the aerodynamic lens developed in this study. However, above $500 \mathrm{~nm}$ the focusing property of the Liu-type lens decreases rapidly, whereas the newly developed lens geometry allows narrow particle beams over a much larger particle size range detectable by the ALABAMA. For the experimentally determined particle beam widths even better focusing properties are obtained in combination with the new lens design. A considerable discrepancy between theoretical and experimentally determined particle beam widths results from particle sizes larger than $1500 \mathrm{~nm}$. Here, the conically shaped orifices in the aerodynamic lens system are probably a major reason for the significantly narrower particle beam widths in the experiments. As already mentioned in the main part, the calculation with the aerosol lens calculator assumes the individual orifices correspond to thin cylindrical discs.

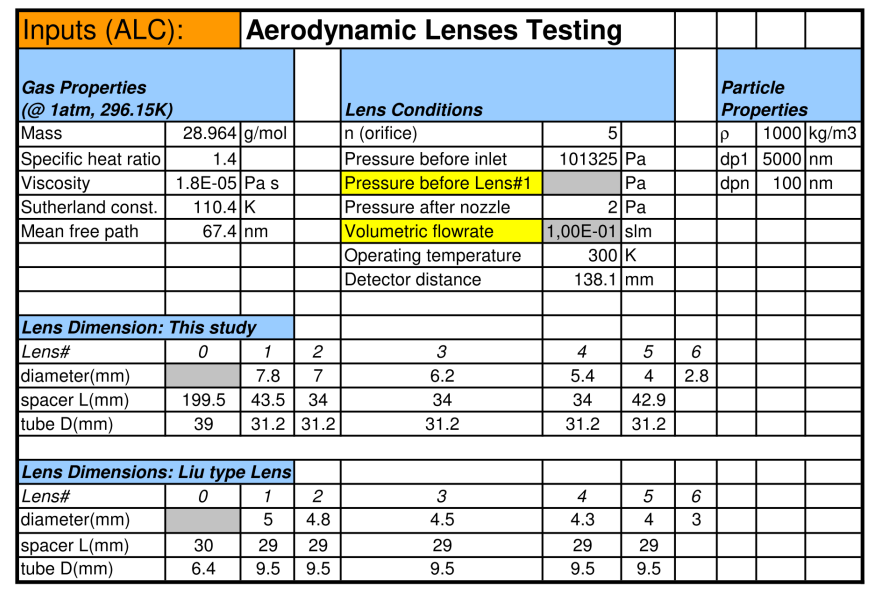

\begin{tabular}{|c|c|c|c|}
\hline \multicolumn{3}{|l|}{ Output (ALC): } & Experimental: \\
\hline $\begin{array}{c}\text { Beam width }(\mathbf{1} \sigma) \text { at } \\
\text { the detector }(\boldsymbol{\mu m})\end{array}$ & Liu type Lens & Lens of this study & Lens of this study \\
\hline $\mathbf{d p}(\mathbf{n m})$ & $\mathbf{2 . ~ D e t e c t o r}$ & 2. Detector & 2. Detector \\
\hline 100 & 103 & 357 & - \\
\hline 150 & 70 & 106 & 133 \\
\hline 200 & 54 & 131 & 102 \\
\hline 300 & 40 & 70 & 67 \\
\hline 400 & 36 & 58 & 51 \\
\hline 500 & 73 & 62 & 33 \\
\hline 600 & 264 & 53 & 41 \\
\hline 800 & 2947 & 69 & 25 \\
\hline 1000 & 3025 & 70 & 25 \\
\hline 1325 & 3017 & 73 & - \\
\hline 1500 & 3012 & 276 & 23 \\
\hline 2000 & 3011 & 2072 & 34 \\
\hline 2550 & 3010 & 6855 & 81 \\
\hline 3000 & 3010 & 13561 & 140 \\
\hline 3775 & 3010 & 19523 & - \\
\hline 5000 & 3009 & 19523 & - \\
\hline & & & \\
\hline
\end{tabular}

Figure S2. A comparison of the results from the calculations of the aerosol lens calculator between the Liu-type lens and the aerodynamic lens from this study. The left table shows the input parameters and the right table shows the resulting size-resolved particle beam widths (in $\mu \mathrm{m})$ for the respective lens design as output. The green marked particle sizes show the particle size range in which the new lens geometry results in a theoretical improved particle beam focusing. In the rightmost column, the particle beam widths experimentally determined in this study are shown for comparison. In order to compare the particle beam widths calculated with the aerosol lens calculator with the particle beam widths determined experimentally, the theoretical values were converted into widths of one sigma. For the conversion of the theoretical values it was assumed that the particle beam diameters originally resulting from the aerosol lens calculator, which comprise $90 \%$ of all particles (Wang and McMurry, 2006a), describe a 2-dimensional circular Gaussian distribution. 


\section{S3 Deflection of charged particles in an electric field}

In order to assess the influence of an electric field on charged particles, their deflection in y-direction is determined theoretically (see Eq. $(\mathrm{S} 2-\mathrm{S} 3)$ ).

40

The force on a charged particle in an electric field is determined by the electric charge of the particles $q$ and the electric field strength $E$ :

$m_{p} \cdot a_{y}=q \cdot E$

The electric field strength corresponds to the ratio of the voltage difference between the positive and the negative electrode $U_{y}$

to the distance between the two electrodes $d_{E x}$ :

$m_{p} \cdot a_{y}=q \cdot \frac{U_{y}}{d_{E x}}$

The integral is formed over the time the particle needs from the entry into the electric field $t_{0}$ to the ablation spot $t$ :

$\int_{t_{0}}^{t} a_{y} \mathrm{~d} t=\frac{q}{m_{p}} \cdot \frac{U_{y}}{d_{E x}} \cdot \int_{t_{0}}^{t} \mathrm{~d} t=v_{y}(t)$

Assuming $t(0)=0$ and $v_{y}\left(t_{0}\right)=0$, the particle velocity $v$ in $\mathrm{y}$-direction at time $t$ results:

$50 \quad v_{y}(t)=\frac{q}{m_{p}} \cdot \frac{U_{y}}{d_{E x}} \cdot t$

To obtain the deflection of the particles $(y)$ in the y-direction at time $t$, the integral over time is formed:

$$
\begin{aligned}
\int_{t_{0}}^{t} v_{y} \mathrm{~d} t & =\frac{q}{m_{p}} \cdot \frac{U_{y}}{d_{E x}} \cdot \int_{t_{0}}^{t} t \mathrm{~d} t \\
y(t) & =\frac{1}{2} \cdot \frac{q}{m_{p}} \cdot \frac{U_{y}}{d_{E x}} \cdot t^{2}
\end{aligned}
$$

Assuming a spherical particle shape, the particle mass can be expressed as a function of particle density $\rho_{p}$ and particle radius $55 r_{p}$ :

$m_{p}=\rho_{p} \cdot V_{p}=\rho_{p} \cdot\left(\frac{4}{3} \cdot \pi \cdot r_{p}^{3}\right)$

If the flight time $t$ is replaced by the ratio of the flight distance $L$ in the electric field (in x-direction) to the particle velocity $v$ in $\mathrm{x}$-direction and the electric charge $q$ is replaced by the product of the elementary-charge constant $e$ and the charge number $z$, the deflection of the particles at the ablation spot results as:

$60 y(t)=\frac{1}{2} \cdot \frac{z \cdot e \cdot U_{y} \cdot L^{2}}{\rho_{p} \cdot\left(\frac{4}{3} \cdot \pi \cdot r_{p}^{3}\right) \cdot d_{E x} \cdot v^{2}}$

Examples of the magnitude of the deflection are given in Sect. 3.2.1 of the main part. 


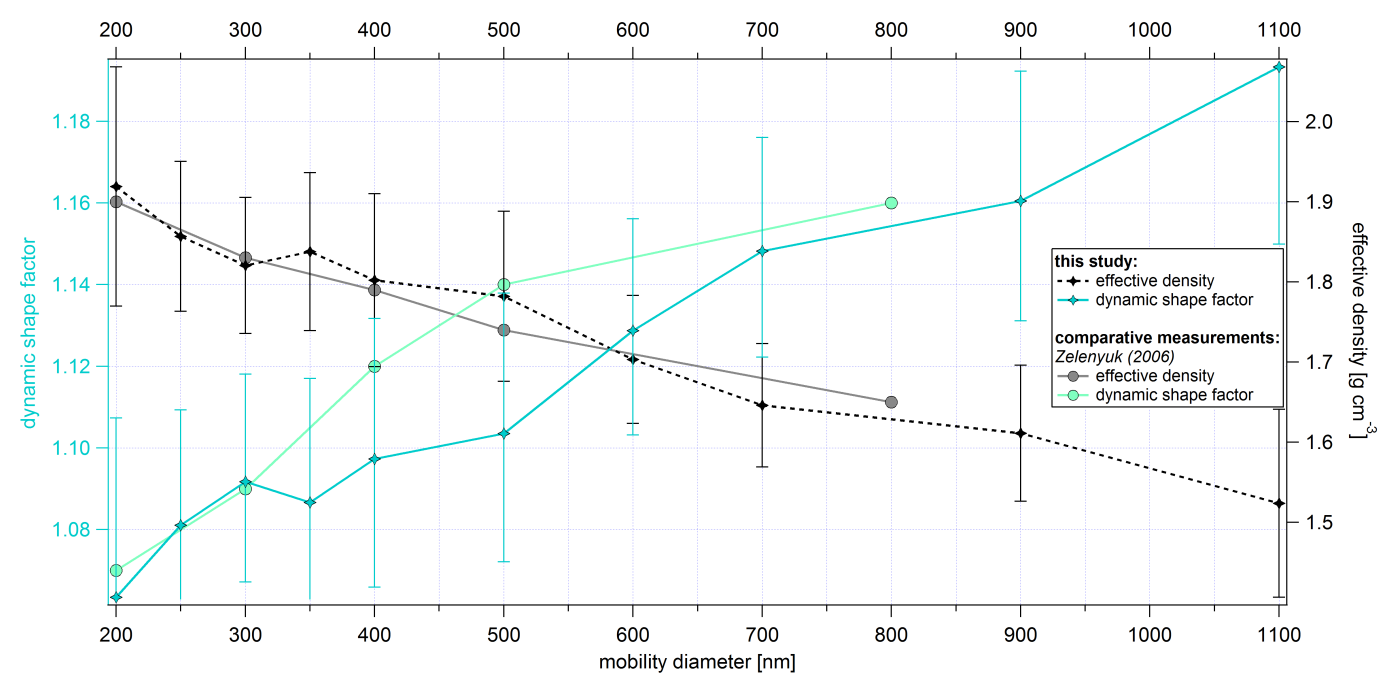

Figure S3. Determination of the size-dependent dynamic shape factors and effective densities for the measured $\mathrm{NaCl}$ particles. The values of the comparison measurement were taken from Zelenyuk et al. (2006). The y-uncertainties result from the standard deviations of the vacuum aerodynamic diameters determined with ALABAMA.

\section{S4 Determination of the $\mathrm{NaCl}$ particle shape}

Using the approach and the assumptions made in DeCarlo et al. (2004) and Zelenyuk et al. (2006), both a dynamic shape factor and an effective density for individual particle types were determined, as shown for $\mathrm{NaCl}$ particles in Fig. S3 using the new ALABAMA setup.

To determine the dynamic shape factor $\bar{X}$ and the effective density $\rho_{e f f}$, the mobility diameter and the vacuum aerodynamic diameter are required according to the following equations $\left(\mathrm{NaCl}: \rho_{p}=2.17 \mathrm{gcm}^{-3}\right)$ :

$$
\begin{aligned}
\bar{X} & =\sqrt{\frac{\rho_{p}}{\rho_{0}} \cdot \frac{d_{m o b}}{d_{v a}}} \\
\rho_{e f f} & =\frac{d_{v a}}{d_{m o b}}
\end{aligned}
$$

The values for the dynamic shape factor and the effective density in Fig. S3 are comparable to the values for $\mathrm{NaCl}$ cubes shown in Zelenyuk et al. (2006), so it was concluded that the $\mathrm{NaCl}$ particles measured in this study have a cubic shape.

\section{S5 Determination of the detection efficiency}

For a conversion of the particle counts per seconds into a particle concentration, the flow into the device, which depends on the lens pressure, is required. The determination of the standard flow into ALABAMA was performed with the TSI flowmeter 4140 or with the DEFINER 220 Dry Cal at lens pressures between $1.5 \mathrm{hPa}$ and $2.7 \mathrm{hPa}$. Afterwards, a polynomial function was fitted to the curve of the lens-pressure-dependent measuring points (see Fig. S4). The uncertainties of the manufacturer $\left( \pm 0.083 \mathrm{~cm}^{3} \mathrm{~s}^{-1}, \mathrm{TSI}\right)$ were used for the additional weighting of the fit. A quadratic fit function $\left(f(x)=K 0+K 1 \cdot x+K 2 \cdot x^{2}\right)$ appears to be useful because the opening cross-section to be passed in the CPI should also have a quadratic dependence. Furthermore, it is assumed that at zero hPa lens pressure the flow into the device must be zero. Accordingly, K0 was set to zero to determine the parameters of the fit function. 


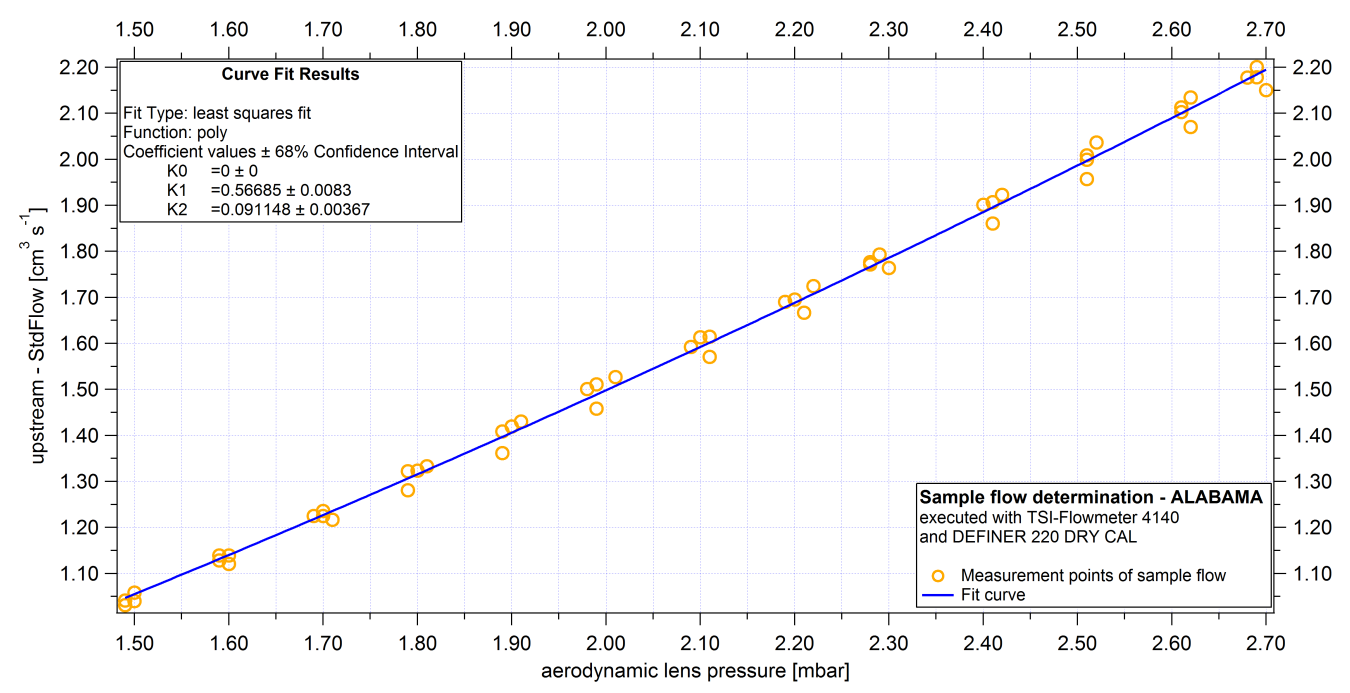

Figure S4. Determination of the lens pressure dependent sample flow into the ALABAMA (see text for details).

To calculate the detection efficiency, the temperature and the pressure in the connected measuring system were used. The temperature information was taken from the flowmeter installed in the particle-free bypass line and the pressure was given by the OPC (see Sect. 4.1.2 and Fig. 9 in the main part). Thus it was possible to convert the standard flow rate (flow Std $_{\text {) into a }}$ volume flow rate $\left(\right.$ flow $_{V}$, in $\left.\mathrm{cm}^{3} \mathrm{~s}^{-1}\right)$, which is needed for a comparison with the OPC and the CPC.

85 flow $_{V}=\frac{\mathrm{flow}_{S t d} \cdot p_{S t d} \cdot \bar{T}}{\bar{p} \cdot T_{S t d}}$

The terms $p_{S t d}$ and $T_{S t d}$ correspond to the standard pressure and standard temperature. The TSI standard conditions are $21.1^{\circ} \mathrm{C}$ and $1013 \mathrm{hPa} . \bar{T}$ and $\bar{p}$ represent the averaged temperature and pressure during the measurement time period. With the help of the volume flow, the averaged number of particles detected per second can be converted into a particle concentration. This allows to determine both the detection efficiency of the individual detection units and those of the sized particles:

$D E_{\text {SizedP }}=\frac{\frac{N_{\text {SizedP }}}{\text { flow }_{V}}}{C_{\text {Ref }}}$

$C_{R e f}$ corresponds to the averaged particle concentration measured at the same time with the reference instruments. $N_{\text {SizedP }}$ is the number of sized particles per second.

\section{S6 Cross talking}

95 Cross talking means if a high signal intensity is detected at one of the two photomultipliers of the detection units, this can cause a "ghost particle count" on the other detection record (the reason for this still has to be found), which would result in an incorrect particle concentration. This effect can be observed mainly at the first and sometimes at the second detection unit in the normal measurement mode. An increase in the detection threshold would lead to a loss of low signals in the edge areas of the laser and thus to a reduction in their effective laser widths. The thresholds were set in particle-free air in such a way that the background noise caused by the cw laser led to almost no erroneous particle counting. At the set thresholds, miscounts 
occured on average every 14 seconds for the first detection unit and every 18 seconds for the second. Incorrect sized particles were only counted every 912 seconds on average. In order to obtain results without cross talking effects, only one of the two detection lasers was used during some of the measurements and the other was switched off accordingly. With this procedure, the measurements were carried out individually for each detection unit. However, in order to measure the sized particles and the hit rate, both detection units had to be switched on simultaneously. Due to the fact that two detectable light scattering signals within a time interval of $(\sim 0.3-1.3 \mathrm{~ms})$ are required for sized particles and due to the limitation of particle concentrations in the characterization measurements, an influence on the number of measured sized particles could not be observed.

\section{S7 Conversion of the motor step size for the tilting of the aerodynamic lens system}

The coefficients resulting from the fit function are initially only output in the step positions of the DC motors. Using calculations from the intercept geometry, a conversion factor can be determined for the step width at the respective measuring position in $\mathrm{x}$-direction. For this, both the geometric distances of the DC motors and the distances of the individual lasers to the pivot point of the aerodynamic lens system must be known. With the respective conversion factors and the knowledge of the step sizes of the DC motors, the results can be given in metric units. An overview of the distances and conversion factors (CF) is given in Fig. S5.

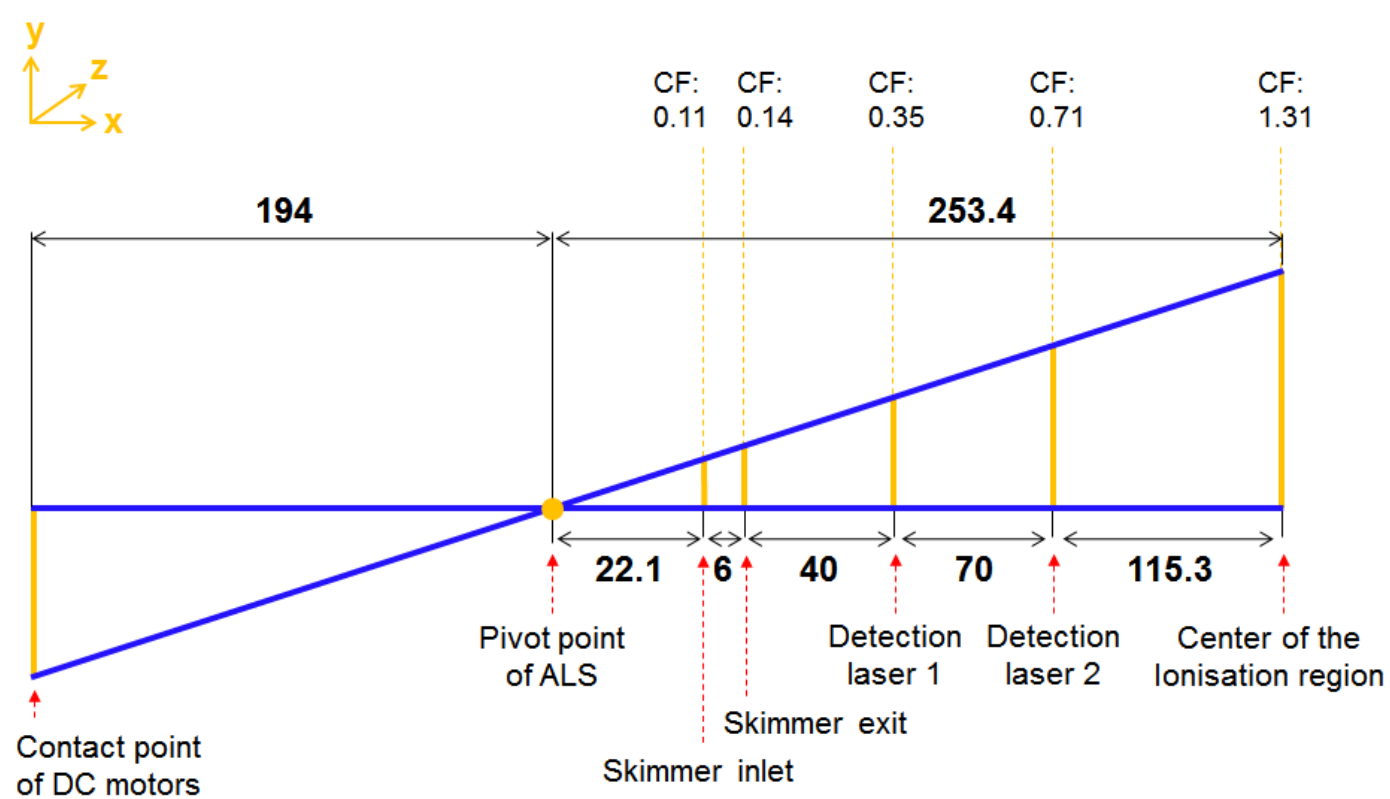

Figure S5. Distances of the components in the ALABAMA (in mm) and the corresponding conversion factors for the lens scan method; (not scaled)

\section{S8 Particle detection rate}

Figure S6 presents the particle detection rate calculated as the product of particle detection efficiency (main part Sect. 4.3.1, Fig. 13), volume flow rate (Sect. S5), for a particle concentration of 1 particle $\mathrm{cm}^{-3}$. A higher flow rate only partly compensates the lower detection efficiency for small particles (below $250 \mathrm{~nm}$ ) with increasing lens pressure, but in general it becomes clear that a higher flow rate increases the particle detection rate. The current configuration limits the lens pressure to $2.6 \mathrm{hPa}$ due to the vacuum requirements for the high voltages. In the future, the lens pressure (and thereby the flow rate) can possibly be 
further increased using a reduced skimmer opening without increasing the pressure in the high voltage area of the vacuum chamber.

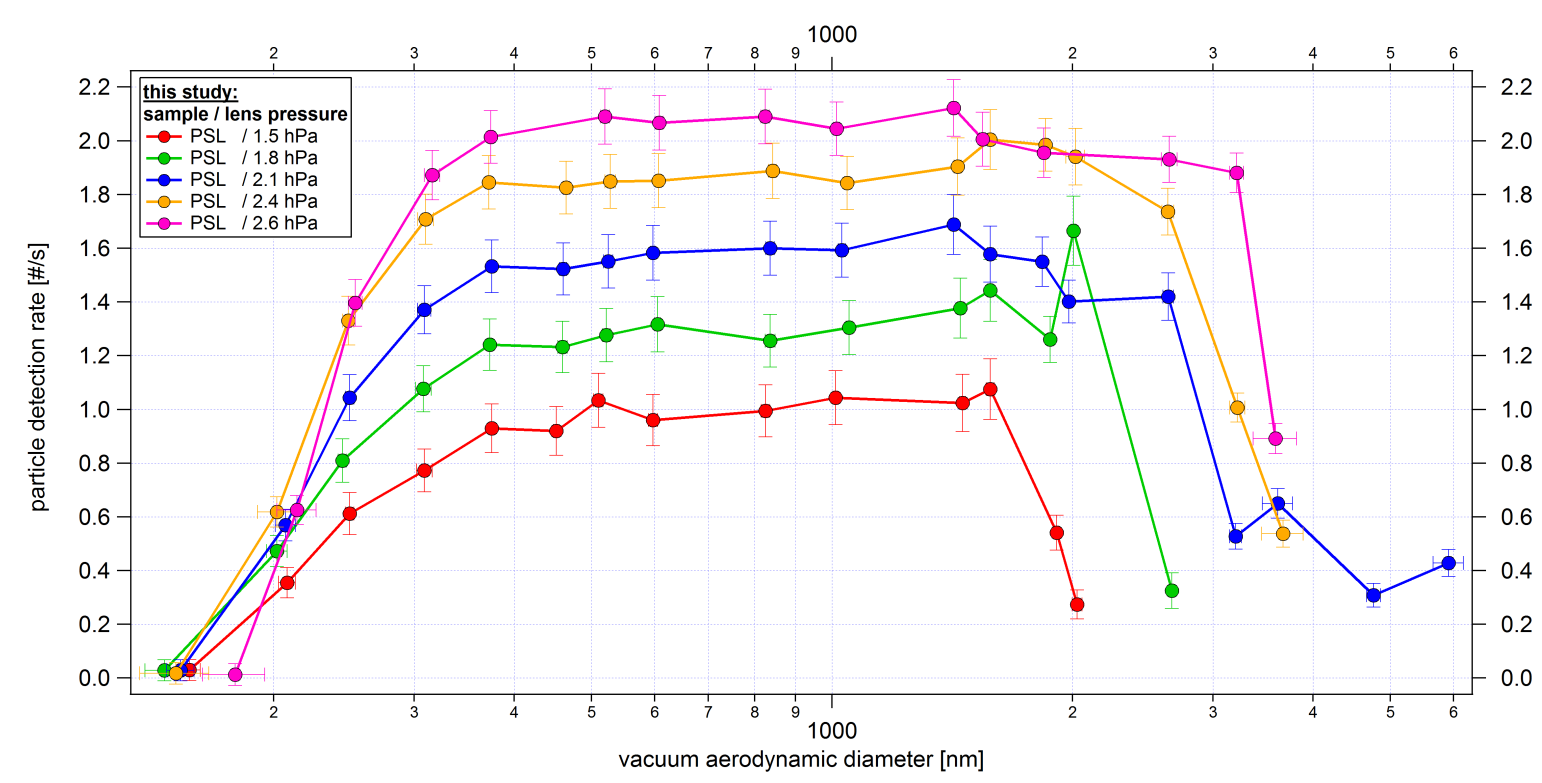

Figure S6. Size-resolved and lens pressure dependent particle detection rate. The y-uncertainty bars are given by the Gaussian error propagation of the detection efficiency and the volume flow rate. The $\mathrm{x}$-uncertainty bars correspond to the standard deviation of the particle size distribution per particle size, particle type and lens pressure measured with ALABAMA, converted into $d_{v a}$ according to Eq. S1

\section{S9 Comparison of particle beam characteristics at a fixed ALS position}

Since no automated lens scan was possible in connection with the previous aerodynamic lens system used by Brands et al. (2011) and Köllner (2020), a qualitative comparison of the particle beam characteristics of the previously used (Köllner, 2020) and the new ALS is presented below. For this purpose, the number of sized particles (particles detected at both detection lasers) are divided by the particle counts recorded at the same time at the first and the second detection unit. Thus, the results essentially depend only on the ALS (+ ALS holder) used and the detection units. Furthermore, uncertainties associated with the external reference devices are therefore irrelevant.

As can be seen in Fig. S7, measurements with the previously used ALS show a similar trend for both detection units, using the number ratio of sized particles $($ SizedP) to particles counted at the two detection stages (DL1 and DL2) as $c 1$ or $c 2$, respectively. The SizedP/c1-curve (DL1) can be explained in that the particles tend to miss the second detection laser more quickly than the first detection laser as the width of the particle beam increases. However, a size-dependent displacement of the particle beam together with the alignment of the ALS specified by the holder or cross talking effects could also be of importance here. For SizedP/ 2 (DL2), a similar curve can be explained by the fact that the second detection laser is aligned perpendicular to the first detection laser. This means that the particles can still be detected at the second detection laser despite missing the first detection laser. This in turn could mean that the particle beam for particles larger than about $900 \mathrm{~nm}$ is broader than the effective width of the first detection laser. Alternatively, the particle beam could be shifted along the beam path of the second laser and thus move out of the first laser at the same time. The same applies to SizedP/c2 for smaller particle sizes. However, the different ratios of $c 1 / c 2$ indicate different causes for the decreasing ratios between smaller and larger sizes. In contrast, measurements with the new ALS show a more similar course between SizedP/c1 and SizedP/c2 over the same size range. In addition, the ratios over a wide size range are over 0.8 . Thus, with the new ALS, the probability that a particle 


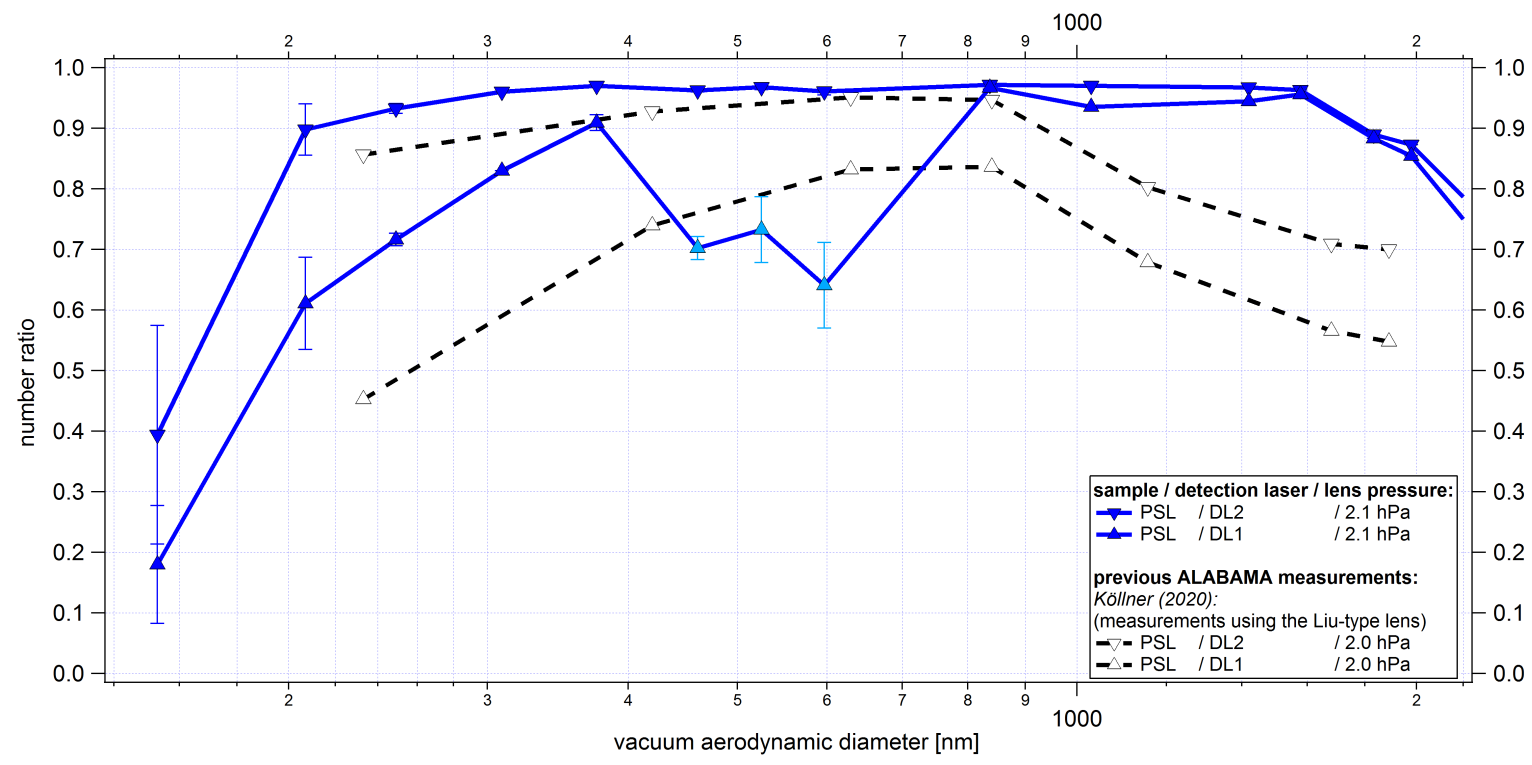

Figure S7. Qualitative comparison of particle beam divergences using the new ALS and the ALS previously used in ALABAMA with the Liu-type lens (Köllner, 2020). The number of sized particles is divided by the number of particles counted at the two detection stages (DL1 and DL2), respectively. The ratio is expected to decrease if the particle beam is wider than the effective width of the detection lasers. The measurement points displayed in a light blue were affected by cross talking effects. The y-uncertainty bars correspond to the standard deviation of the ratios.

(PSL) triggers a signal at both detection lasers is higher. This applies to both smaller and larger particles. On the one hand, this could be explained by a narrower particle beam across all sizes or by a smaller size-dependent displacement of the particle beam. The outliers between about $400 \mathrm{~nm}$ and $600 \mathrm{~nm}$ that can be observed at the first detection unit are due to cross talking effects, the cause of which could not be definitively clarified. Regardless of whether the differences between the previously used and the new ALS are caused by size-dependent particle beam widths or size-dependent displacements of the particle beam, the aforementioned indicators suggest that the new ALS achieves improved particle focusing.

\section{S10 Multiple charge correction}

When using $\mathrm{NaCl}$ particles smaller than $600 \mathrm{~nm}\left(d_{v a}\right)$, additional corrections were made to determine the detection efficiencies and hit rates. The reason for this is that below $600 \mathrm{~nm}$ not only singly charged but also multiply charged particles passed through the DMA, albeit only in low concentrations due to the impactor used. Thus, for the analysis an additional size-dependent selection was applied. For this purpose, the size-resolved UHSAS measurements were used. The corrected detection efficiency can be determined by selecting the corresponding size bins from the UHSAS and ALABAMA (according to Eq. S1). With this correction, for example, the detection efficiency of $\mathrm{NaCl}$ particles of size $464 \mathrm{~nm}\left(d_{v a}\right)$ was adjusted from $3.8 \%$ to $2 \%$ in Sect. 4.3.1 (main part), whereas the particle number in the second size mode in the UHSAS is only about $3 \%$ of the particle number in the size mode with the singly charged $\mathrm{NaCl}$ particles.

\section{S11 Determination of stick spectra and units of measure}

First, a wavelet transformation (Mexican hat wavelet) is performed to de-noise the raw spectra and correct the baseline. The positive part of the wavelet should then correspond to the measured signals (Klimach, 2012). Subsequently, a mass calibration 
over all mass spectra is performed with known ion masses (further details in Klimach, 2012). The resulting positions of the mass-to-charge ratios serve as starting points for the determination of the ion peak areas. For this purpose, a range around the respective mass-to-charge ratio is determined by means of a predetermined signal width, into which the raw signal is integrated over time. The averaged background level (defined by the height of the raw signal a half $\mathrm{m} / \mathrm{z}$ position before and behind the respective mass-to-charge ratio) is then subtracted. The result is the so-called stick spectra. The height of a single stick for a specific mass-to-charge ratio thus corresponds to the ion peak area in $\mathrm{mV}^{*}$ sample (sample: temporal resolution of the oscilloscope in $2 \mathrm{~ns}$ ) for a mass-to-charge ratio and is referred to in this study as the $\mathrm{m} / \mathrm{z}$ signal intensity.

\section{S12 Size dependent characterization of mass spectral signals}

To study the size dependence of the mass spectral signals, only the highest values (summed intensities and number of $\mathrm{m} / \mathrm{z}$ signals) were selected from each scan (as shown in Fig. 21 of the main part). These maximum values were averaged for measurements with different charge numbers (from $z=1$ to $z=5$ ) at this size, whereby the respective scan positions of the maximum values of the curves were not taken into account. Figure S8 shows the particle size resolved differences between the three setups for the cation $\mathrm{m} / \mathrm{z}$-signals.

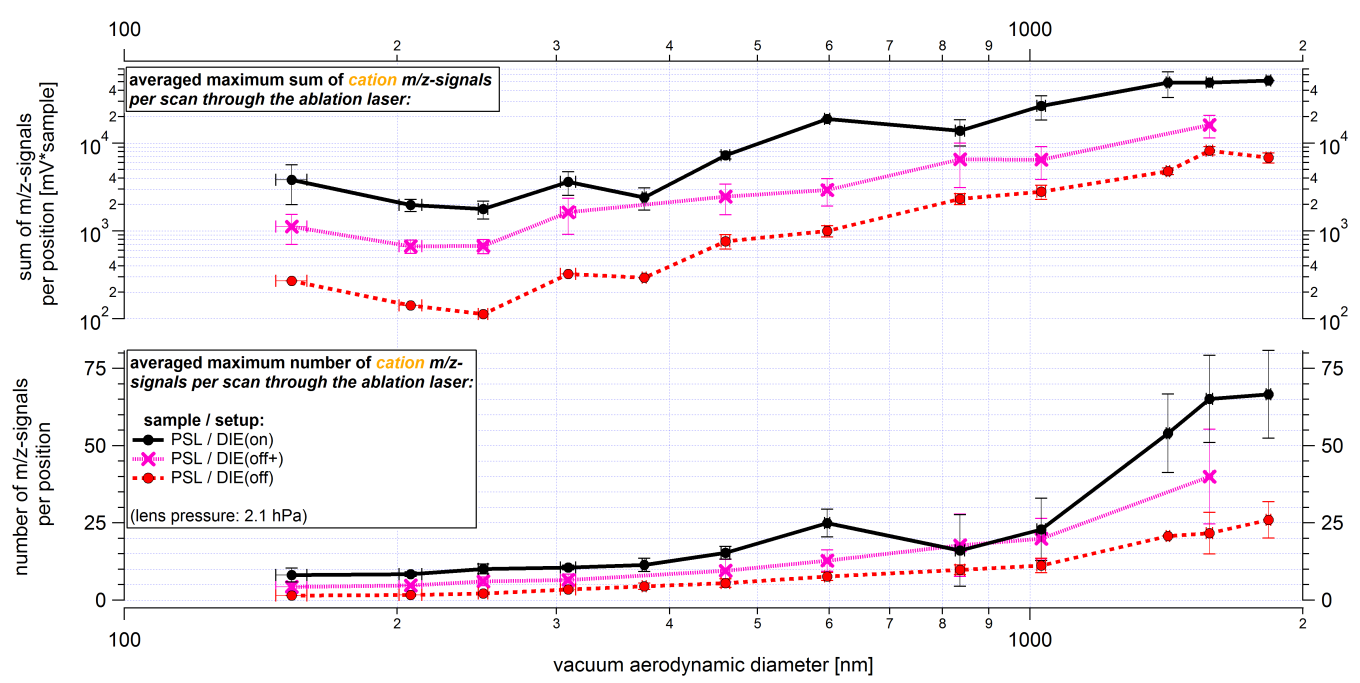

Figure S8. Size-resolved mass spectral information of cation $\mathrm{m} / \mathrm{z}$-signals in dependence of the ion extraction field setup. The upper panel shows the curve for the respective maximum signal intensities per lens scan (according to the approach shown in Fig. 21 of the main part). The lower panel shows the curve for the maximum number of $\mathrm{m} / \mathrm{z}$-signals per lens scan. Measurements were performed with laser energies between 4.8 and $5.4 \mathrm{~mJ}$ and DMA selected particle charge numbers from 1e+ up to 5e+. The y-uncertainty bars correspond to the standard deviation of the number and sum of the m/z-signals of the mass spectra. The x-uncertainty bars correspond to the standard deviation of the particle size distribution per particle size, particle type and lens pressure measured with the ALABAMA, converted into $d_{v a}$ according to Eq. S1

First, there is a clear enhancement in intensity (upper panel at Fig. S8) and number (lower panel at Fig. S8) of cation signals using the delayed ion extraction compared to the setups DIE(off) and DIE(off+). Only for the particle sizes $837 \mathrm{~nm}$ and $1029 \mathrm{~nm}$ similar results can be observed in the number of $\mathrm{m} / \mathrm{z}$-signals using the DIE(off+) setup. The reason for this is not known. For all other particle sizes there is a clear separation between the results of both setups. As a result, there are more $\mathrm{m} / \mathrm{z}$-signals (cations) together with higher signal intensity resulting from using the delayed ion extraction.

In contrast to the cation signals, a comparison of the results from using the DIE(on) setup and DIE(off + ) setup shows a very similar behavior in Fig. S9. Considering the sum of the $\mathrm{m} / \mathrm{z}$-signals or their number, differences can only be detected at the lower end or at the upper end of the size range. However, there is a big discrepancy to the DIE(off) setup. In this case anion 


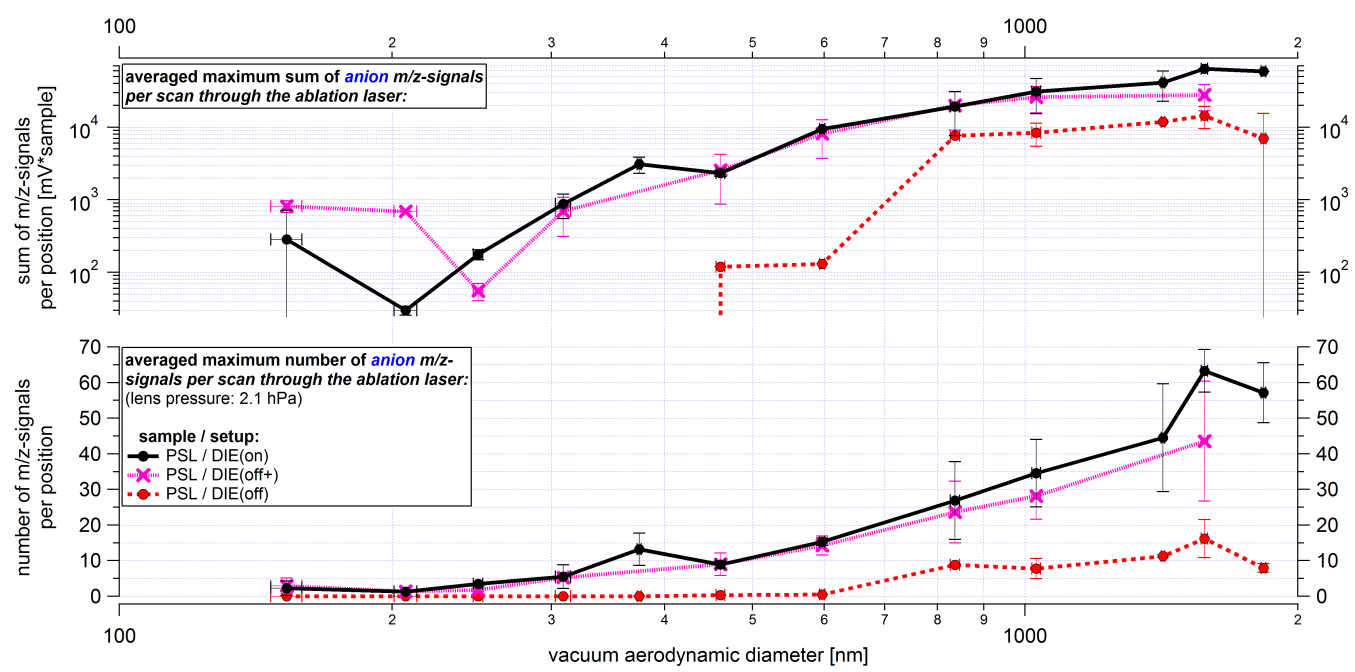

Figure S9. Caption the same as for Fig. S8, except for anions.

signals can only be detected for particle sizes above $460 \mathrm{~nm}\left(d_{v a}\right)$. However, the detected signal intensities and the number of $\mathrm{m} / \mathrm{z}$-signals are significantly below those of the two other setups.

\section{S13 Reproducibility of mass spectral signals at a fixed ALS position}

To check whether the DIE also has a advantageous effect on the reproducibility of mass spectra, the relative dispersion of the number of $\mathrm{m} / \mathrm{z}$-signals was investigated. For this purpose, the respective number of the $\mathrm{m} / \mathrm{z}$-signals of cations and anions were formed for 100 randomly selected individual particle mass spectra. The lower and upper quartiles of the number of the $\mathrm{m} / \mathrm{z}$-signals were used to measure the dispersion. The difference between the upper and lower quartiles was divided by the median, which gives a relative dispersion based on the median (approach modified according to Drewnick, 2000). To define the existence of a signal, the threshold value of $\bar{A} \pm 3 \cdot \sigma$ was applied to the stick spectrum. To reduce the effect of charge-dependent particle deflection, only particle samples larger than $400 \mathrm{~nm}$ and with a charge number of $\mathrm{z}=1$ were used.

Figure S10 shows that in the case of cations, the use of the DIE tends to lead to a slight improvement in the relative dispersion of the number of $\mathrm{m} / \mathrm{z}$-signals. The increase in dispersion towards larger particle sizes can be related to a shift in the particle beam. If the particle beam is shifted to the area of the falling flanks of laser intensity, a larger variation of fragmentation may occur (as can be seen from the standard deviation in Fig. 21 of the main part). In the case of anions, on the other hand, no clear tendencies or improvements are discernible. Nevertheless, the advantage of using the delayed ion extraction becomes clear from the analysis of the cation signals.

\section{S14 Mass spectral analysis of ice nucleating particles on the Birch pollen sample}

The evaluation of the birch pollen washing water (referred as Birch pollen) mass spectra indicates that contamination might play a role for the different hit rates with and without prior ice activation, as presented in Fig. 23 of the main part. Therefore, we first applied the fuzzy-c-means algorithm (Roth et al., 2016; Hinz et al., 1999; Bezdek et al., 1984) to the mass spectra of the particle sample experiments to check for different particle types, which are represented as clusters of mass spectra. Briefly, the algorithm was started with twenty randomly chosen mass spectra as starting points for the cluster centers. After that, every mass spectrum was correlated with the mass spectra (cluster centers) of the twenty clusters and assigned to the cluster with the highest correlation coefficient. The resulting cluster mean mass spectra of the Birch pollen sample are comparable to 


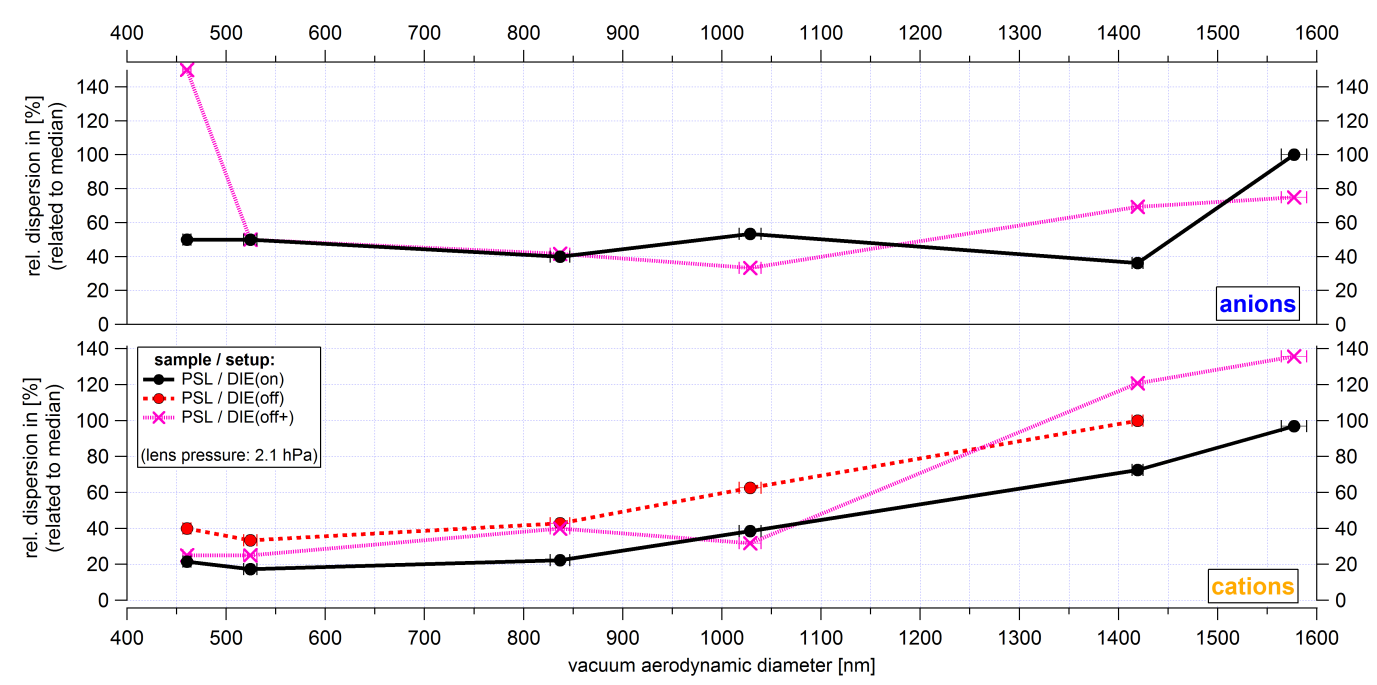

Figure S10. Reproducibility of mass spectral signals (upper panel: anion and lower panel: cation) in dependence of the ion extraction field setups. The x-uncertainty bars correspond to the standard deviation of the particle size distribution per particle size, particle type and lens pressure measured with ALABAMA, converted into $d_{v a}$ according to Eq. S1

those found by Schmidt (2016), although both the Birch pollen and the Sunflower pollen spectra shown there can be found in our data set. Based on the characteristic signals (ion markers) of the clusters a further analysis of the mass spectra was performed, the ion marker method (see Köllner, 2020, for more details). For this method, the ion markers must exceed a threshold value which has been determined by means of empty spectra as described in Sect. 4.5.5 (main part). Separate criteria had to be established for both polarities due to the partial absence of anion or cation signals. Typical biological ion marker for this interpretation include organic nitrogen and phosphorus: $C N(-26), C N O(-42), C_{3} H_{3} O_{2}(-71), P_{3}(-79), P O(+47)$ (Creamean et al., 2014). Further ion marker that could be assigned to birch pollen included $K(+39)$ and $K_{2} C N(+104)$. In contrast to the fuzzy-c-means algorithm, the ion marker method is exactly reproducible and does not depend on randomly chosen mass spectra as starting points for the cluster centers.

215 Figure S11 shows the relative abundance of the particle types found in the six pollen experiments using the ion marker method. The gray colored particle types represent particles that can be attributed to biological origin like Birch pollen. Although it was not to be expected, particle compositions of mineral or metallic origin with signals of lithium, silicon oxide, aluminium, aluminosilicate, chromium and iron were found in addition to the mass spectra assigned to the Birch pollen. Furthermore, in some mass spectra, both mineral and biological signals were found together in the particles, hereinafter referred to as BioMin particles. It is shown that the mineral/metallic and BioMin particles make up a higher percentage of the total fraction during measurements with ice activation compared to measurements without ice activation, no matter if FINCH or SPIN is used. The difference in the FINCH measurement with ice activation can be attributed to the increased ion yield and larger effective width of the ablation laser when using the DIE(on) setup compared to the DIE(off) setup as described in Sect. 4.5.4 (main part). In general, it was not possible to clarify the origin of the mineral/metallic particle type within the scope of this work.

If the particles would indeed be due to contamination, this could result in a different hit rate, since mineral or metal containing particles have different shapes and chemical compositions compared to birch pollen, which can be assumed to be almost spherical (based on images under the microscope, Pummer et al., 2012; Augustin et al., 2013). The particle shape is decisive for particle focusing within the ALS. Further, the chemical elements of the contaminants can be ionized differently with the ablation laser, which has an influence on the ion yield and thus also on the hit rate. The assumption of a worse focusability/ionization of the mineral dust or metal containing particles compared to the birch pollen would therefore lead to a decrease 


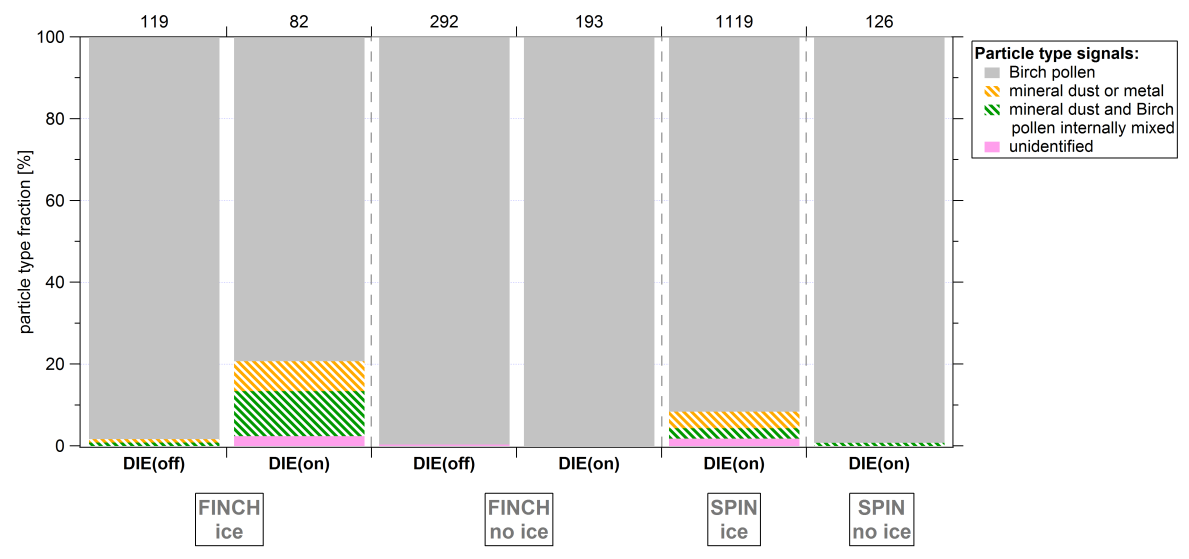

Figure S11. Particle type fractions using birch pollen sample as an example and using different measurement setups: FINCH or SPIN together with PCVI in ice activation mode (labeled as ice): with prior ice activation and separation process; FINCH or SPIN together with PCVI in no ice activation mode (labeled as no ice): without prior ice activation and separation process, but still with the particle flow through FINCH or SPIN and PCVI. For the Birch pollen sample the SPIN measurements were only performed with the DIE(on) setup. The unidentified particle type contains all those particles whose signals cannot be assigned to any of the other three particle types. The number of generated mass spectra for each experiment is given on top of the graph.

of the hit rate if such contamination particles are present. Nevertheless, the following calculations suggest that contamination cannot be the main cause of the reduced hit rate:

$H R_{\text {ice }}=H R_{\text {no ice }}(P S) \cdot n \operatorname{Frac}(P S)+H R($ cont $) \cdot n F r a c($ cont $)$

where $n F r a c$ is the number fraction of the particle sample $(P S)$ or of the contaminants (cont), $H R_{i c e}$ is the resulting hit rate with prior ice activation, $H R_{\text {no ice }}(P S)$ is the hit rate of the particle sample without prior ice activation, and $H R($ cont $)$ is the hit rate of the contaminants. The highest percentage of the desired particle sample, i.e. $n F r a c(P S)$, and thus the lowest percentage of the contamination, i.e. $n$ Frac(cont), we get for the worst acceptable hit rate of the contamination. Since there can be no negative hit rates, this would be $H R($ cont $)=0$. Under these assumptions and using the results from Fig. 23 (Sect. 5, main part), the following number fractions result:

\section{Birch pollen:}

FINCH and DIE(off): $\mathrm{nFrac}(\mathrm{PS})=0.34+\mathrm{nFrac}($ cont $)=0.66$

FINCH and DIE(on): $\mathrm{nFrac}(\mathrm{PS})=0.73+\mathrm{nFrac}($ cont $)=0.27$

SPIN and DIE(on): $\mathrm{nFrac}(\mathrm{PS})=0.61+\mathrm{nFrac}($ cont $)=0.39$

\section{Feldspar:}

SPIN and DIE(on): $\mathrm{nFrac}(\mathrm{PS})=0.60+\mathrm{nFrac}($ cont $)=0.40$

This would be an immensely high contamination rate, which is actually even higher, since at least for DIE(on) the contamination hit rate is $>0$ as Fig. S1 1 shows, where contamination was detected. Since this could not be seen in the number size distribution measurements behind the PCVI, it should therefore be excluded as a reason for the reduced hit rate at "ice" with prior ice activation and separation process. 


\section{S15.1 Uncertainties of the detection efficiency}

The measurement uncertainty of the detection efficiency is determined as follows,

$\sigma_{D E}=\frac{C_{A L A}}{C_{R e f}} \sqrt{\left(\frac{1}{\sqrt{N_{A L A}}}\right)^{2}+\left(\frac{1}{\sqrt{N_{R e f}}}\right)^{2}+\left(\frac{\sigma_{\text {flow }}}{\text { flow }_{\text {Std }}}\right)^{2}+\left(\frac{\sigma_{p}}{p}\right)^{2}+\left(\frac{\sigma_{T}}{T}\right)^{2}+\left(\frac{\sigma_{C_{R e f}}}{C_{R e f}}\right)^{2}} \cdot 100$

where $C_{A L A}$ is the particle concentration calculated from the ALABAMA measurements. $C_{A L A}$ can be both the calculated particle concentrations at the first or second detection unit, but also those of the sized particles. $C_{R e f}$ corresponds to the particle concentration measured with the reference instruments (OPC, CPC). $N_{A L A}$ and $N_{R e f}$ are the particle counts measured with each instrument, which are required to determine the relative statistical uncertainties. The third term indicates the relative uncertainty of the standard sample flow into ALABAMA. The fourth and fifth terms represent the relative uncertainties of the measured pressure and temperature, which are given by the manufacturers with $2 \%$ and $1 \%$. The last term indicates the uncertainties of the reference devices (Grimm OPC 1.129: $3 \%$ and TSI CPC 3010: 10\%).

\section{S15.2 Uncertainties of the flow determination}

The polynomial fit function $f(x)=K 0+K 1 \cdot x+K 2 \cdot x^{2}$ (parameters see Fig. S4) and gaussian error propagation is used to determine the uncertainties of the sample flow $\sigma_{\text {flow }}$ (Eq. S10), into ALABAMA. A $2.5 \%$ uncertainty of the differential pressure sensor used (Analog Microelectronics) and a variation range of the lens pressure of $0.02 \mathrm{hPa}$ are assumed.

$$
\begin{aligned}
& \sigma_{\text {flow }} a=\sqrt{\left(\left(K 1 \cdot p_{L}+K 2 \cdot p_{L}^{2}\right)-\left(K 1 \cdot\left(p_{L}+0.02\right)+K 2 \cdot\left(p_{L}+0.02\right)^{2}\right)\right)^{2}} /\left(K 1 \cdot p_{L}+K 2 \cdot p_{L}^{2}\right) \\
& \sigma_{\text {flow }} b=\sqrt{\left(\left(K 1 \cdot p_{L}+K 2 \cdot p_{L}^{2}\right)-\left((K 1+\sigma K 1) \cdot p_{L}+(K 2+\sigma K 2) \cdot p_{L}^{2}\right)\right)^{2}} /\left(K 1 \cdot p_{L}+K 2 \cdot p_{L}^{2}\right) \\
& \sigma_{\text {flow }} c=\sqrt{\left(\left(K 1 \cdot p_{L}+K 2 \cdot p_{L}^{2}\right)-\left(K 1 \cdot\left(p_{L}+p_{L} \cdot 0.025\right)+K 2 \cdot\left(p_{L}+p_{L} \cdot 0.025\right)^{2}\right)\right)^{2}} /\left(K 1 \cdot p_{L}+K 2 \cdot p_{L}^{2}\right)
\end{aligned}
$$

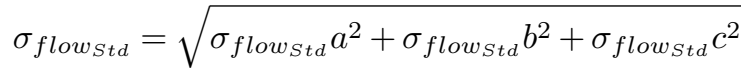

In addition, the uncertainties ( $\sigma K 1$ and $\sigma K 2$ ) of the polynomial fit function is taken into account.

\section{S15.3 Uncertainties of the hit rate}

To determine the uncertainty of the hit rate $\sigma_{H R}$, the approach presented in Köllner (2020) is used.

$\sigma_{H R}=\frac{\sqrt{N_{H i t s} \cdot(1-H R)}}{N_{\text {Shots }}}$

The uncertainty of the hit rate (HR) is thus determined on the basis of binomial statistics related to the number of laser pulses from the ablation laser $N_{\text {Shots }}$ and the number of successfully detected mass spectra $N_{H i t s}$ resulting from these laser pulses.

\section{S15.4 Uncertainties of the particle beam divergences}

To determine the uncertainty of the particle beam divergence $\sigma_{P B D}$ the uncertainties of the particle beam widths at the first $\sigma \frac{}{P B W_{D L 1}}$ and second $\sigma_{\overline{P B W_{D L 2}}}$ detection lasers are used.

$\sigma_{P B D}=\sqrt{\left(\frac{\sigma_{\overline{P B W_{D L 2}}}}{7}\right)^{2}+\left(\frac{\sigma_{\overline{P B W_{D L 1}}}}{7}\right)^{2}}$ 
Since measurements at one particle size were usually performed several times, the standard errors of the mean of the particle 285 beam widths were used for these measurement points. The nominal distance of $7 \mathrm{~cm}$ between the two detection lasers is taken from Brands et al. (2011). 


\section{References}

Augustin, S., Wex, H., Niedermeier, D., Pummer, B., Grothe, H., Hartmann, S., Tomsche, L., Clauss, T., Voigtländer, J., Ignatius, K., and Stratmann, F.: Immersion Freezing of Birch Pollen Washing Water, Atmospheric Chem. Phys., 13, 10989-11003, https://doi.org/10.5194/acp-13-10989-2013, 2013.

Bezdek, J. C., Ehrlich, R., and Full, W.: FCM: The Fuzzy c-Means Clustering Algorithm, Computers \& Geosciences, 10, 191-203, https://doi.org/10.1016/0098-3004(84)90020-7, 1984.

Brands, M., Kamphus, M., Böttger, T., Schneider, J., Drewnick, F., Roth, A., Curtius, J., Voigt, C., Borbon, A., Beekmann, M., Bourdon, A., Perrin, T., and Borrmann, S.: Characterization of a Newly Developed Aircraft-Based Laser Ablation Aerosol Mass Spectrometer (ALABAMA) and First Field Deployment in Urban Pollution Plumes over Paris During MEGAPOLI 2009, Aerosol Sci. Technol., 45, 46-64, https://doi.org/10.1080/02786826.2010.517813, 2011.

Creamean, J. M., Lee, C., Hill, T. C., Ault, A. P., DeMott, P. J., White, A. B., Ralph, F. M., and Prather, K. A.: Chemical Properties of Insoluble Precipitation Residue Particles, J. Aerosol Sci., 76, 13-27, https://doi.org/10.1016/j.jaerosci.2014.05.005, 2014.

DeCarlo, P. F., Slowik, J. G., Worsnop, D. R., Davidovits, P., and Jimenez, J. L.: Particle Morphology and Density Characterization by Combined Mobility and Aerodynamic Diameter Measurements. Part 1: Theory, Aerosol Sci. Technol., 38, 1185-1205, https://doi.org/10.1080/027868290903907, 2004.

Drewnick, F.: Entwicklung und Charakterisierung eines Laser-massenspektrometrischen Verfahrens: Ein Beitrag zur Aerosolanalytik, PhD Thesis, Universität Hohenheim, 2000.

Hinz, K. P., Greweling, M., Drews, F., and Spengler, B.: Data Processing in On-Line Laser Mass Spectrometry of Inorganic, Organic, or Biological Airborne Particles, J. Am. Soc. Mass Spectrom., 10, 648-660, https://doi.org/10.1016/S1044-0305(99)00028-8, 1999.

Klimach, T.: Chemische Zusammensetzung der Aerosole : Design und Datenauswertung eines EinzelpartikelLaserablationsmassenspektrometers, PhD Thesis, Johannes Gutenberg-Universität Mainz, 2012.

Köllner, F.: Aerosol particles in the summertime arctic lower troposphere: chemical composition, sources, and formation, PhD Thesis, Johannes Gutenberg-Universität Mainz, 2020.

310 Pummer, B. G., Bauer, H., Bernardi, J., Bleicher, S., and Grothe, H.: Suspendable Macromolecules Are Responsible for Ice Nucleation Activity of Birch and Conifer Pollen, Atmospheric Chem. Phys., 12, 2541-2550, https://doi.org/10.5194/acp-12-2541-2012, 2012.

Roth, A., Schneider, J., Klimach, T., Mertes, S., van Pinxteren, D., Herrmann, H., and Borrmann, S.: Aerosol Properties, Source Identification, and Cloud Processing in Orographic Clouds Measured by Single Particle Mass Spectrometry on a Central European Mountain Site during HCCT-2010, Atmos. Chem. Phys., 16, 505-524, https://doi.org/10.5194/acp-16-505-2016, 2016.

315 Schmidt, S.: Analyse Der Chemischen Zusammensetzung von Eis-Und Wolkenresiduen Mittels Einzelpartikel-Massenspektrometrie Und Charakterisierung von Aerosolpartikeln Aus Anthropogenen Und Natürlichen Quellen, PhD Thesis, Johannes Gutenberg-Universität Mainz, 2016.

Wang, X. and McMurry, P. H.: A Design Tool for Aerodynamic Lens Systems, Aerosol Sci. Technol., 40, 320-334, https://doi.org/10.1080/02786820600615063, 2006a.

Wang, X. and McMurry, P. H.: Instruction Manual for the Aerodynamic Lens Calculator, Aerosol Sci. Technol., 40, 1-10, https://doi.org/10.1080/02786820600616764, 2006b.

Zelenyuk, A., Cai, Y., and Imre, D.: From Agglomerates of Spheres to Irregularly Shaped Particles: Determination of Dynamic Shape Factors from Measurements of Mobility and Vacuum Aerodynamic Diameters, Aerosol Sci. Technol., 40, 197-217, https://doi.org/10.1080/02786820500529406, 2006. 